\title{
lodinated Contrast Prior to Evaluation for Thyrotoxicosis
}

Brian D. Phillips, mo ${ }^{1}$ James V. Hennessey, mo ${ }^{2}$

\author{
${ }^{1}$ Division of Endocrinology, Warren Alpert School of Medicine at Brown University, Providence, Rhode Island. \\ ${ }^{2}$ Division of Endocrinology, Beth Israel Deaconess Medical Center, Harvard Medical School, \\ Boston, Massachusetts.
}

BACKGROUND: Radioactive iodine is useful in the diagnosis and treatment of patients with newly diagnosed thyrotoxicosis. However, in many patients these tests are not possible to obtain due to recent administration of iodinated contrast for other types of diagnostic studies, most frequently computed tomography (CT) scans.

OBJECTIVE: To determine the frequency of iodinated contrast use in hospital inpatients newly diagnosed with thyrotoxicosis and the utility of these studies.

DESIGN: Retrospective chart review.

SETTING: Academic medical center.

INTERVENTION: None.

PATIENTS: Inpatients with newly diagnosed thyrotoxicosis who were seen in consultation by the endocrinology service. MEASUREMENTS: The records of inpatient endocrinology consultations $(\mathrm{n}=1171)$ performed by our service over a 4-year period were reviewed. Records of patients with newly diagnosed thyrotoxicosis were identified and reviewed to determine if iodinated contrast had been recently administered, the types of studies it was used for, and the results of those studies.

RESULTS: Forty-five patients (45\%) had been given iodinated contrast within 2 weeks prior to endocrinology evaluation, 43 for CT scanning, and 2 for angiography. Of the patients who underwent CT scanning, 7 (16\%) had a finding that potentially changed their inpatient management. Only 1 of these patients required emergent treatment of a condition diagnosed by CT scan before further diagnostic studies could have been performed.

CONCLUSIONS: A substantial number of inpatients with thyrotoxicosis receive iodinated contrast prior to endocrinologic evaluation. Acute findings from these studies that change patient management are uncommon. Awareness of this issue may facilitate care of hospitalized patients with thyrotoxicosis. Journal of Hospital Medicine 2009;4:285-288. @ 2009 Society of Hospital Medicine.

KEYWORDS: contrast, CT scan, hyperthyroidism, iodine, thyrotoxicosis.

Thyrotoxicosis is a relatively common endocrine disorder. An epidemiologic study estimated the prevalence of hyperthyroidism in the United States at $1.3 \% .^{1}$ In our experience newly diagnosed thyrotoxicosis is 1 of the more frequent reasons for inpatient endocrinology specialty consultation. Generally, the diagnosis of thyrotoxicosis is made on clinical and laboratory grounds, and consultation is obtained in order to assist with diagnosing the specific cause and to arrange necessary treatment. A 24-hour radioactive iodine uptake and scan are useful studies for differentiating the cause of thyrotoxicosis. These tests provide an indication of thyroid pathophysiology based on measurement and imaging of thyroidal uptake of a small dose of orally administered radioiodine. In thyrotoxic patients, the uptake is typically high in those with conditions associated with increased hormone synthesis, such as Graves' disease or autonomously functioning thyroid nodules, and is low in those with conditions associated with decreased hormone synthesis, such as thyroiditis or exogenous thyroid hormone ingestion.

Establishing the correct diagnosis has significant impact on the selection of appropriate therapy for these vastly different etiologies. Radioactive iodine in the form of I-131 is useful in the treatment of certain thyrotoxic conditions. Radioiodine is the treatment most frequently employed in the United States for Graves' disease, ${ }^{2}$ and is also the most commonly used treatment of solitary toxic nodules and toxic multinodular goiter. However, it is of no use in treatment of subacute thyroiditis or exogenous ingestion of thyroid hormone. While antithyroid drugs such as methimazole or propylthiouracil can be used to treat conditions associated with increased thyroid hormone synthesis, they also are not effective in treating subacute thyroiditis or thyrotoxicosis factitia; therefore, it is important to establish the diagnosis before using these medications.

Exogenous iodine that has been administered prior to administration of radioiodine can interfere with the uptake of radioiodine by the thyroid, thus rendering the 24-hour uptake and scan inaccurate as a means of diagnosis, and interfering with the utility of I-131 as a treatment. ${ }^{3}$ In our experience, many inpatients with newly diagnosed thyrotoxicosis receive exogenous iodine in the form of intravenous (IV) contrast agents near the time of their presentation, leading to an inability to utilize radioactive iodine to diagnose and potentially treat them. One common reason for the use of iodinated contrast is to enhance computed tomography (CT) scan images. Many symptoms that could presumably lead to the ordering of a CT scan may overlap with symptoms of thyrotoxicosis. Prompt diagnosis of 
thyrotoxicosis, along with awareness of the interference of IV contrast with use of radioactive iodine and the frequency with which this occurs, may prevent unnecessary CT scans in some patients. Therefore, we undertook this study to quantify the frequency of this occurrence, and the frequency that the IV contrast studies were ultimately useful in the management of these patients.

\section{Methods}

The records of inpatient endocrinology consultations over a 48-month period (January 2003 through January 2007) were reviewed. Consultations requested for thyroid disease were identified, and those which were for thyrotoxicosis were reviewed. Patients with thyrotoxicosis with a cause that had been previously determined, or who had already undergone treatment, were not included in the analysis. The remaining patients with new onset thyrotoxicosis, based on clinical and laboratory findings, in whom radioactive iodine would have been useful for diagnostic and potentially treatment purposes, were included.

Of the patients included, our records were reviewed to determine demographic data, whether intravenous contrast had been administered within 2 weeks of endocrinology consultation, and the type of study that was performed. The results of the contrast studies were reviewed, with attention to findings that potentially could have changed acute or inpatient management of the patients.

\section{Results}

A total of 1171 consults were reviewed. Of these, 324 $(27.7 \%)$ were for thyroid disease. One hundred patients (8.5\% of total consults, $30.8 \%$ of thyroid disease consults) were identified with previously unevaluated thyrotoxicosis as the primary reason for the consult. Of these patients, $74 \%$ were women, and $26 \%$ were men. The mean age was 54.6 years (range 18-93 years).

Forty-five patients (45\%) had been given iodinated contrast within 2 weeks prior to endocrinology evaluation, 43 for CT scanning, and 2 for angiography. There were a total of 50 contrast-enhanced CT scans done (7 patients had CT scans of multiple anatomic regions). There were 26 chest CT scans, 16 abdominal/pelvic CT scans, and 8 neck CT scans. Indications for the CT scans in these patients are shown in Table 1.

Of the 43 patients who underwent CT scanning, 7 (16\%) had a finding that potentially changed their inpatient management ( 2 with tracheal compression by a goiter, 1 with airway compromise from tonsillar edema, 1 with appendicitis, 1 with pulmonary hypertension, 1 with diffuse lymphoma, and 1 with a rib fracture after trauma). Only 1 of these patients (appendicitis) required emergent treatment of their condition before further diagnostic studies could have been reasonably performed. Among the angiography patients, 1 had undergone cardiac catheterization, which was diagnostic of coronary artery disease, and 1 had under-

\begin{tabular}{ll} 
TABLE 1. Indications for CT Scans & \\
Indication for CT Scan & Number of Patients \\
\hline Palpitations/shortness of breath & 20 \\
Abdominal pain & 6 \\
Neck swelling & 6 \\
Weight loss & 2 \\
Atrial fibrillation & 1 \\
Loose stool & 1 \\
Question of abdominal mass & 1 \\
Jaundice & 1 \\
Palpable lymphadenopathy & 1 \\
Trauma & 1 \\
Abnormal chest radiograph & 1 \\
Unknown & 2 \\
\hline
\end{tabular}

gone angiography of the lower extremities, which revealed an acute arterial clot.

Also of note, in April 2005 at our institution, a new emergency department opened which made it logistically easier to obtain a CT scan. Prior to that time, 16 of 49 patients (33\%) evaluated for thyrotoxicosis had received iodinated contrast for a CT scan. After that time, 27 of 51 patients (52\%) received a contrast CT study.

\section{Discussion}

Indications for contrast-enhanced CT scans in the acute care setting are numerous. The majority of the CT scans ordered in the above patients were of the chest. CT scans of the chest may be used to evaluate suspected pulmonary emboli or aortic dissection. Coronary CT angiography is also being studied as a method of risk stratification of patients presenting with acute chest pain. ${ }^{4}$ Common signs and symptoms of the above disorders include chest pain, dyspnea, palpitations, and tachycardia. Many of the most common cardiovascular signs and symptoms associated with thyrotoxicosis overlap with those seen in the above disorders. A recent study of thyrotoxic patients found that on presentation, $73 \%$ had palpitations, $60 \%$ had dyspnea, $25 \%$ had chest pain, and $35 \%$ had cough. ${ }^{5}$ Mean heart rate has been shown to be significantly elevated in thyrotoxic patients compared with normal controls. ${ }^{5,6}$ Atrial fibrillation or flutter can occur in about $8 \%$ of thyrotoxic patients, especially in males, in older ages, and in those with underlying cardiac disease. $^{7}$

We also noted that 16 out of the 50 total contrastenhanced CT scans performed were of the abdomen and pelvis. While abdominal and gastrointestinal signs and symptoms seem to be less common manifestations of thyrotoxicosis than cardiovascular ones, hyperdefecation with or without diarrhea can be seen in approximately in one-third of cases. Nausea, vomiting, abdominal pain, and jaundice occur less commonly but can be seen occasionally with severe thyrotoxicosis. ${ }^{3}$ Weight loss is common, and fever may occur occasionally, which also may trigger evaluation by imaging for malignancy or infection. 
Indications for the CT scans ordered for the patients in this study can be seen in Table 1 . Some of the studies were ordered for symptoms not likely to be thyroid-related and may have been unavoidable. However, many of the studies, especially those done for complaints such as palpitations, dyspnea, and weight loss were likely obtained due to symptoms that ultimately turned out to be caused by thyrotoxicosis.

A variety of other factors may uncommonly decrease radioactive iodine uptake, including cardiac decompensation, vomiting after ingestion of the orally dosed radioiodine capsule, and medications, including thioureas. However, exogenous iodine is probably the most common interfering factor. $^{8}$ Exogenous iodine decreases thyroidal radioiodine uptake both by dilution of the total body iodine pool, and by inhibition of thyroid hormone synthesis via the WolffChaikoff effect. ${ }^{8}$ Sources of iodine include diet, medications (amiodarone, which contains approximately $6 \mathrm{mg}$ of inorganic iodine per $200 \mathrm{mg}$ tablet, and potassium iodide), and, most prominently in the hospitalized patient, intravenous contrast material. It has been shown that exposure to as little as $100 \mu \mathrm{g}$ of intravenous iodide can suppress uptake in hyperthyroid patients. ${ }^{9}$ Both ionic and nonionic intravenous contrast media contain sufficient amounts of inorganic iodide $(\sim 10 \mu \mathrm{g} / \mathrm{ml})$ to induce this suppression when given in typical doses of $50-200 \mathrm{ml}$. This iodide may be derived from deiodination of the contrast media molecule, and also from free iodide contaminants. ${ }^{10}$ The duration of uptake suppression varies among patients and generally ranges from 4 to 12 weeks, although it may be shorter in hyperthyroid patients. ${ }^{8}$ This long-term suppression may be related to the slow deiodination of contrast media remaining in the body. ${ }^{10}$ Urinary iodine normalization can be used as a means of determining the time when uptake can be measured without interference from exogenous iodine.

Iodine administration can also have deleterious effects on thyroid function apart from its effect on radioiodine uptake. In euthyroid patients, exogenous iodine in large doses inhibits organification of iodide and thyroid hormone synthesis (Wolff-Chaikoff effect). Normally this effect diminishes after several weeks, but in patients with autoimmune thyroid disease, it may persist, leading to hypothyroidism. ${ }^{11}$ Iodine ingestion may also lead to hyperthyroidism. In areas of endemic iodine deficiency, this is thought to represent unmasking of thyroid autonomy that had been suppressed by lack of iodine, encompassing such patients as those with Graves' disease or autonomous nodules. In areas of iodine sufficiency such as the United States, the incidence of iodine-induced thyrotoxicosis is low, and typically occurs in patients with autonomous thyroid nodules or multinodular goiter. $^{11,12}$ Cases of thyroid storm have been reported following iodinated contrast administration. ${ }^{13}$ The elderly may be at greater risk for iodine-induced thyrotoxicosis, ${ }^{14}$ which is of concern given their inherent higher likelihood of cardiac disease. It is possible that in some of the patients in our study who had CT scans done for nonthyroid-related symptoms, iodinated contrast could have precipitated subsequent thyrotoxicosis. In general, caution should be used when administering intravenous contrast agents to patients with known thyroid disease.

CT scanning is being used much more frequently in the acute care setting. One study in the emergency department at a single institution revealed that from the years 2000 to 2005 , CT scanning of the chest increased by $226 \%$, and of the abdomen by $72 \%$, despite only a $13 \%$ increase in patient volume and a stable level of acuity. ${ }^{15}$ We refer the reader to this recent review for a discussion of the recent increase in the use of CT scans and the associated risks. ${ }^{16}$ We have found that a substantial proportion of inpatients with thyrotoxicosis receive intravenous contrast prior to endocrinologic evaluation, thus limiting the ability to use radioactive iodine in diagnosis and treatment. The vast majority of these diagnostic studies are contrast-enhanced CT scans. Acute findings from these studies in this population that change patient management are uncommon.

The increasing usage of CT scans may exacerbate this problem. Education of physicians that are likely to diagnose thyrotoxicosis prior to subspecialty evaluation (internists, family practitioners, and emergency medicine physicians) regarding the similarity of thyrotoxic symptoms to those typically triggering the request of a CT scan is essential. Clinicians should have an appreciation of the interference of iodinated contrast with the ability to obtain a radioiodine uptake and scan. They should also be aware of the potential effects of iodinated contrast on thyroid function when ordering a contrast-enhanced CT scan in patients with known thyroid disease or symptoms consistent with the presence of thyrotoxicosis. Under these circumstances consideration of thyroid dysfunction with appropriate blood tests may result in more accurate and timely diagnosis and treatment of underlying thyroid disease and enhance patient outcomes.

\section{Address for correspondence and reprint requests:}

James V. Hennessey, MD, Beth Israel Deaconess Medical Center, Division of Endocrinology, 330 Brookline Avenue, Boston, MA 02215; Telephone: (617) 667-9344; Fax: (617) 667-7060; E-mail: Jhenness@bidmc.harvard.edu Received 29 October 2007; revision received 4 February 2008; accepted 21 March 2008.

\section{References}

1. Hollowell JG, Staehling NW, Flanders WD, et al. Serum TSH, T , and thyroid antibodies in the United States population (1988 to 1994): National Health and Nutrition Examination Survey (NHANES III). J Clin Endo Metab. 2002;87:489-499.

2. Wartofsky L, Glinoer D, Solomon B, Lagasse R. Differences and similarities in the diagnosis and treatment of Graves' disease in Europe, Japan, and the United States. Thyroid. 1991;1:129-135.

3. Davies TF, Larsen PR.Thyrotoxicosis. In:Larsen PR, Kronenberg HM, Melmed S, Polonsky KS, eds. Williams Textbook of Endocrinology. 10th ed. Philadelphia: Saunders; 2003:374-421.

4. White CS. Chest pain in the emergency department. JThorac Imaging. 2007;22:49-55.

2009 Society of Hospital Medicine DOI 10.1002/jhm.384 Published online in wiley InterScience (www.interscience.wiley.com). 
5. Osman F, Franklyn JA, Holder RL, et al. Cardiovascular manifestations of hyperthyroidism before and after antithyroid therapy. J Am Coll Cardiol. 2007;49:71-81.

6. Klein I, Ojamaa K. Thyroid hormone and the cardiovascular system. N Engl J Med. 2001;344:501-509.

7. Frost L, Vestergaard P, Mosekilde L. Hyperthyroidism and risk of atrial fibrillation or flutter. Arch Intern Med. 2004; 164:1675-1678.

8. Grayson RR. Factors which influence the radioactive iodine thyroidal uptake test. Am J Med. 1960;28:397-415.

9. Childs DS, Keating FR, Rall JE, et al. The effect of varying quantities of inorganic iodide (carrier) on the urinary excretion and thyroidal accumulation of radioiodine in exophthalmic goiter. J Clin Invest. 1950;29:726-738.

10. Laurie AJ, Lyon SG, Lasser EC. Contrast material iodides: potential effects on radioactive iodine thyroid uptake. J Nucl Med. 1992;33:237-238.
11. Roti E, Ettore U. Iodine excess and hyperthyroidism. Thyroid. 2001;11: 493-500.

12. Molen AJ, Thomsen HS, Morcos SK. Effect of iodinated contrast media on thyroid function in adults. Eur Radiol. 2004;14:902-907.

13. Weber C, Scholz GH, Lamesch P, Paschke R. Thyroidectomy in iodine induced thyrotoxic storm. Exp Clin Endocrinol Diabetes. 1999;107: 468-472.

14. Martin FI, Deam DR. Hyperthyroidism in elderly hospitalized patients. Med J Aust. 1996;164:200-203.

15. Broder J, Warshauer DM. Increasing utilization of computed tomography in the adult emergency department, 2000-2005. Emerg Radiol. 2006;13: 25-30.

16. Brenner DJ, Hall EJ. Computed tomography-an increasing source of radiation exposure. $N$ Engl J Med. 2007;357: 2277-2284. 